\title{
GlyT2+ Neurons in the Lateral Cerebellar Nucleus
}

\author{
Marylka Uusisaari • Thomas Knöpfel
}

Published online: 14 October 2009

(C) The Author(s) 2009. This article is published with open access at Springerlink.com

\begin{abstract}
The deep cerebellar nuclei (DCN) are a major hub in the cerebellar circuitry but the functional classification of their neurons is incomplete. We have previously characterized three cell groups in the lateral cerebellar nucleus: large non-GABAergic neurons and two groups of smaller neurons, one of which express green fluorescence protein (GFP) in a GAD67/GFP mouse line and is therefore GABAergic. However, as a substantial number of glycinergic and glycine/GABA co-expressing neurons have been described in the DCN, this classification needed to be refined by considering glycinergic neurons. To this end we took advantage of a glycine transporter isoform 2 (GlyT2)eGFP mouse line that allows identification of GlyT2expressing, presumably glycinergic neurons in living cerebellar slices and compared their electrophysiological properties with previously described DCN neuron populations. We found two electrophysiologically and morphologically distinct sets of GlyT2-expressing neurons in the lateral cerebellar nucleus. One of them showed electrophysiological similarity to the previously characterized GABAergic cell group. The second GlyT2+ cell population, however, differed from all other so far described
\end{abstract}

M. Uusisaari $\cdot$ T. Knöpfel $(\bowtie)$

Laboratory for Neuronal Circuit Dynamics,

Brain Science Institute, RIKEN,

2-1 Hirosawa,

Wako-shi, Saitama 351-0198, Japan

e-mail: tknopfel@brain.riken.jp

Present Address:

M. Uusisaari

Theoretical and Experimental Neurobiology Unit, OIST,

12-22 Suzaki,

Uruma-shi, Okinawa 904-2234, Japan neuron types in DCN in that the cells (1) are intrinsically silent in slices and only fire action potentials upon depolarizing current injection and (2) have a projecting axon that was often seen to leave the DCN and project in the direction of the cerebellar cortex. Presence of this so far undescribed DCN neuron population in the lateral nucleus suggests a direct inhibitory pathway from the DCN to the cerebellar cortex.

Keywords Cerebellum · Cerebellar nuclei · GlyT2 . Glycinergic neuron · In vitro

\section{Introduction}

Glycinergic neurons, glycine transporters, and glycine receptors are widely expressed in the cerebellum [1-3]. A particular feature of the glycinergic transmission in the deep cerebellar nuclei (DCN) is that despite the wide expression of glycine receptors (glyR) throughout the development and presence of glyR-mediated synaptic transmission in neonatal (postnatal day 7-10) and young adult ( $>17$ day old) rat DCN, no glycinergic synaptic events can be evoked in the time window between postnatal days 13 to P17 $[4,5]$.

We recently described three types of DCN neurons, based on gamma-aminobutyric acid decarboxylase isoform 67 (GAD67) expression and electrophysiological properties [6]. These groups include the large non-GABAergic neurons ("GADnL"; putatively corresponding to the glutamatergic projection neurons) and two classes of smaller, probably local neurons, one of which expresses GAD67 and is thus GABAergic ("GAD+") and the other which is GAD67-negative ("GADnS"). All of these cells consistently fired spontaneously even in the absence of synaptic 
input. However, this categorization was limited in that it did not clarify which of the described cell types would correspond to the glycinergic neurons of the DCN. Especially intriguing was the identity of the GADnS cells, which were found to be local non-GABAergic neurons.

Previous studies [1, 7-9] have concluded that the GABAergic and glycinergic neurons of the DCN are partially overlapping neuronal populations, and that the glycinergic neurons in DCN are predominantly of small-tomedium size $(<20 \mu \mathrm{m}[1,9])$. Thus a reasonable hypothesis was that (1) at least part of the GAD+ cells would be glycine/GABA co-expressing local neurons and (2) the GADnS cells would correspond to the purely glycinergic local neurons.

To test these hypotheses we took advantage of a bacterial artificial chromosome transgenic mouse line expressing enhanced green fluorescence protein (eGFP) under the control of the glycine transporter type 2 (GlyT2) gene promoter [10]. The GlyT2 is responsible for glycine accumulation in neurons [11] and is a specific marker for glycinergic neurons $[2,12]$. Specifically, no glial cells or non-glycinergic neurons have been found to express GlyT2 in the adult DCN [2]. Patch-clamp recordings from eGFPexpressing neurons in the GlyT2-eGFP mice (20 or more days old) revealed that a proportion of them were similar to the previously described GAD + cells in their spontaneous and evoked firing properties whereas a distinct population of the GlyT2+ cells expressed a resting membrane potential below firing threshold and irregular firing unlike all other cell types previously described in the DCN. In addition, morphological examination of this distinct population of GlyT2+ cells revealed that unlike the other GlytT2+ neurons and the previously described GAD + neurons that are characterized by a local axon, their axons leave the vicinity of the cell body and project outside the DCN towards the cerebellar cortex, where they could sometimes be followed up to the granule cell layer. We conclude that inactive GlyT2+ cells represent a previously undescribed DCN neuron population that gives rise to a putatively inhibitory nucleocortical pathway.

\section{Methods}

\section{Slice Preparation}

All experimental protocols were approved by the RIKEN Experimental Animal Committee and conducted in compliance with the Guidelines for the Use of Animals in Neuroscience Research (The Society for Neuroscience, Washington, DC, USA). Young adult (postnatal days 2028) GlyT2/GFP [10] or GAD67/GFP mice [13] were anesthetized with halothane and decapitated. The cerebel- lum was quickly removed and mounted for sectioning with a vibratome (Leica VT1200S, Leica Microsystems, Nussloch, Germany) in ice-cold standard physiological solution [artificial cerebrospinal fluid (ACSF)] containing (in $\mathrm{mM}$ ): $\mathrm{NaCl}, 124$; $\mathrm{KCl}, 3 ; \mathrm{KH}_{2} \mathrm{PO}_{4}, 1.2 ; \mathrm{MgSO}_{4}, 1.9$; glucose, $20 ; \mathrm{NaHCO}_{3}$, 26; and $\mathrm{CaCl}_{2}, 2$; and gassed with $95 \% \mathrm{O}_{2}-5 \% \mathrm{CO}_{2}$. The two to three coronal slices $(300 \mu \mathrm{m})$ containing the DCN were allowed to recover for $>1 \mathrm{~h}$ in oxygenated ACSF at room temperature $\left(21-23^{\circ} \mathrm{C}\right)$ and used for recording during the next $4 \mathrm{~h}$.

\section{Electrophysiological Recordings}

Slices were transferred to a submerged-type chamber mounted on a Leica DMLFSA microscope equipped with differential interference contrast (DIC) optics and wholefield epifluorescence (Till Photonics, Gräfelfing, Germany) imaging. Slices were superfused with gassed ACSF (2$3 \mathrm{ml} / \mathrm{min}$ ), at room temperature $\left(23-24^{\circ} \mathrm{C}\right)$. Borosilicate glass-patch electrodes (outer diameter $2 \mathrm{~mm}, 4-7 \mathrm{MOhm}$ ) were filled with intracellular solution containing (in $\mathrm{mM}$ ) K-gluconate, 140; $\mathrm{NaCl}, 10$; HEPES, 10; EGTA, 0.2; MgATP, 4; Na-GTP, 0.4; glutathione, 5; and biocytin, 8, pH adjusted to 7.3 with $\mathrm{KOH}(280 \mathrm{mOsm})$. The junction potential (calculated value, $14.5 \mathrm{mV}$ ) was not corrected for.

Cell-attached voltage-clamp and whole-cell patch-clamp recordings in current-clamp mode were acquired and controlled using the Axon 700B Multiclamp amplifier and pClamp acquisition software (Molecular Devices, Union City, CA, USA). The sampling frequency was $10 \mathrm{kHz}$. All the recorded cells were in the lateral nucleus.

In the beginning of each recording the input resistance and time constant were measured using hyperpolarizing current steps small enough so that the $I_{\mathrm{h}}$-like voltage sag was not resolved, and from these values the apparent membrane capacitance $\left(C_{\mathrm{m}}\right)$ was estimated. All subsequent amplitudes of current steps were normalized on $C_{\mathrm{m}}$, so that one current step increment amounted to $0.3 \mathrm{pA} / \mathrm{pF}$.

Electrophysiological characterization was concluded within 5-10 min after breaking into whole-cell mode but the whole-cell configuration was kept for more than $20 \mathrm{~min}$ to ensure sufficient biocytin fill by diffusive loading through the patch pipette. The electrode was then gently withdrawn from the cell body. Slices were kept for an additional hour in room temperature ACSF to provide sufficient time for biocytin diffusion into distal dendrites and axons and then fixed in $4 \%$ paraformaldehyde in $0.1 \mathrm{M}$ phosphate-buffered saline (PBS, $\mathrm{pH} 8$ ) at $4^{\circ} \mathrm{C}$ for $12 \mathrm{~h}$.

Measurement of Electrophysiological Parameters

For action potential (AP) shape analysis, several tens of consecutive APs were peak-aligned and averaged. This 
averaged waveform was used to measure AP parameters as follows. For AP half-width we used the AP duration at halfamplitude. AP threshold was defined as the $V_{\mathrm{m}}$ measured $0.5 \mathrm{~ms}$ before the peak in the second derivative of the waveform. AP peak $V_{\mathrm{m}}$ was the voltage at highest point of the AP (cells with non-overshooting APs were discarded). Afterhyperpolarization (AHP) peak was measured at the first local minimum in the waveform after the AP peak, and AHP peak time was the time of this minimum in relation to the AP peak time. For each cell type the grand average of these measures was calculated along with the average AP waveform. The inherently silent cells were driven to fire at $\sim 10 \mathrm{~Hz}$ with depolarizing current, whereas for the spontaneously active cells the AP shape was examined without bias current. For frequency-current (f-I) measures, the mean firing frequency evoked with each increasing current step was measured; for frequency adaptation, the inter-spike intervals of APs evoked in response to a current step that evoked $\sim 30 \mathrm{~Hz}$ firing were measured. Maximum firing frequency was measured as the inverse of the shortest interspike interval during the step depolarization protocols using the strongest depolarization that resulted in the cell firing throughout the step. Jitter in firing regularity was measured for individual inter-spike intervals (ISI) during the same depolarizing steps as the absolute difference (in $\mathrm{ms}$ ) between consecutive ISIs.

\section{Biocytin Histochemistry}

Fixed slices (4\% paraformaldehyde, $\mathrm{pH}$ 7.4) were washed with PBS and endogenous peroxidases were quenched by 5-min incubation in $1 \% \mathrm{H}_{2} \mathrm{O}_{2}$. Tissue was reacted overnight in avidin-biotin complex (ABC Elite kit; Vector Laboratories, Burlingame, CA, USA) at $4^{\circ} \mathrm{C}$. Finally, biocytin was demonstrated by 3,3'-diaminobenzidine tetrahydrochloride histochemistry. Slices with DAB-stained cells were mounted on objective glass under coverslip and digitally imaged using a Leica DC500 digital camera on a Leica DMRE microscope. Whole $z$ axis stack sequence of the biocytin-filled cells was projected into a single image using Image Pro Plus 5.1 software (Media Cybernetics, Silver Spring, MD, USA) and the cell body area was calculated as the area of the cross-section of the cell body in the image. Soma length was measured as the longest diameter of the cell body. Dendritic distance was measured as the longest distance between a visible dendrite tip and soma center. The branching index (BI) was determined by dividing the number of dendritic branches crossing a $30-\mu \mathrm{m}$-radius circle centered on the soma by the number of primary dendrites visible. All spatial features were analyzed using ImagePro Plus 5.1 software. Immunohistochemical demonstration of GFP-expressing cells in the GlyT2-eGFP mouse was performed as described previously [14].

\section{Data Analysis}

Electrophysiological data were analyzed using Clampfit 9.2 software (Molecular Devices, Union City, CA, USA).

Statistical analysis of all parameters was performed using OriginLab 7 software (OriginLab, Northampton, MA, USA). Because no differences over the studied age range were seen in the measured parameters, the data from cells at all ages were pooled. All data are presented as means $\pm \mathrm{SE}$; for statistical significance, Student's $t$ test or analysis of variance (ANOVA) was used where applicable. Differences were considered significant at $p<0.05$.

\section{Results}

Identification of Glycinergic Cells in the DCN

We used a line of transgenic mice in which enhanced green fluorescent protein (eGFP) is faithfully expressed under the GlyT2+ promoter [10]. As GlyT2 is considered a reliable marker for glycinergic cells in adult DCN [2, 10, 12], we identify eGFP-expressing cells as presumably glycinergic in these mice. In the lateral nucleus these presumed glycinergic cells were of various sizes and scattered throughout the nucleus (Fig. 1 (A)). In living slices the eGFP fluorescence was very bright, allowing for easy identification of the GlyT2+ cells for patch clamping. Examples of DIC and epifluorescence views of different-sized eGFP-expressing cells in the lateral nucleus are shown in Fig. 1 (B).

\section{Spontaneous Activity and Action Potential Shapes} of Glycinergic Cells in the DCN

DCN neurons typically fire spontaneously both in intact animals, in slice preparations, and as isolated cells [15-17]. Previously, we showed that the spontaneous firing frequency differs between cell types and especially the large nonGABAergic (GADnL) neurons fire significantly faster than the GABAergic $(\mathrm{GAD}+)$ or small non-GABAergic (GADnS) cells [6]. However, even the slower-spiking GABAergic cells expressed robust spontaneous firing behavior, seen both in external cell-attached and wholecell current clamp recordings with no bias current.

Surprisingly, out of the 38 eGFP-expressing neurons of all sizes recorded in GlyT2/GFP+ mice, aged P20-28, a large number (16 out of 38 ) did not show spontaneous action potentials at a rate exceeding $0.1 \mathrm{~Hz}$, even in the presence of gabazine, a $\mathrm{GABA}_{\mathrm{A}}$-receptor antagonist $(n=3$; data not shown). In some of these cells external recording revealed action potentials at a very low and irregular rate $(<0.1 \mathrm{~Hz}$; Fig. 1 (Ci)) whereas others appeared to be entirely silent. Also, when the whole-cell configuration was 


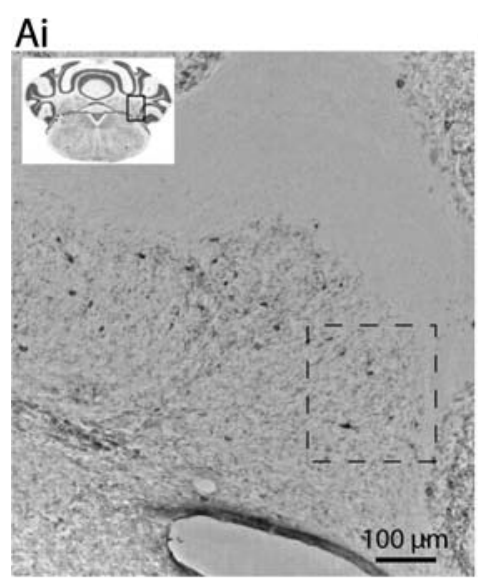

Aii

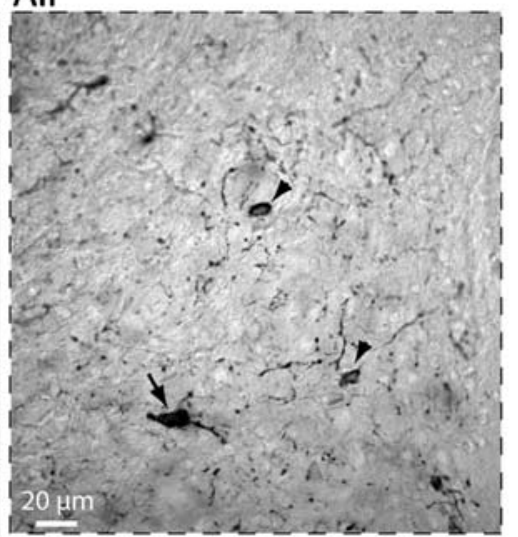

$\mathrm{Bi}$
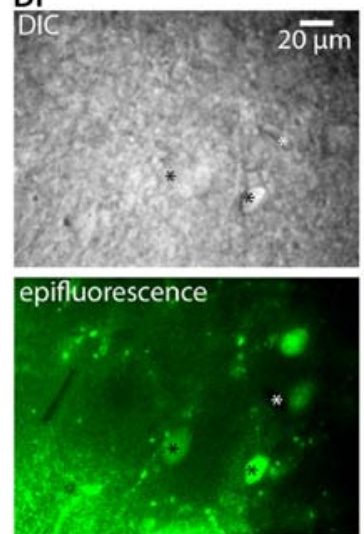

Bii

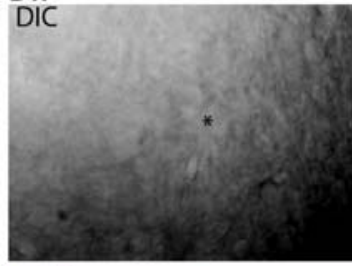

epifluorescence
$\mathrm{Ci}$ Cell-attached voltage-clamp

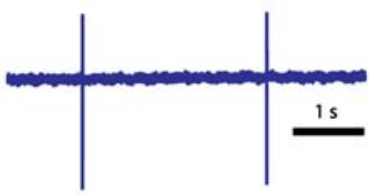

$\mathrm{Di}$

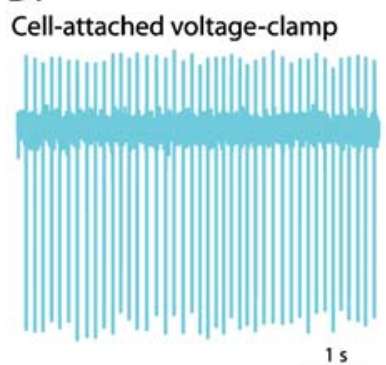

Cii

Whole-cell current-clamp

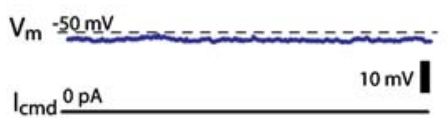

Dii

Whole-cell current-clamp

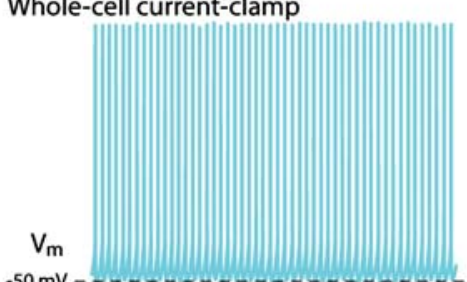

$10 \mathrm{mv}$

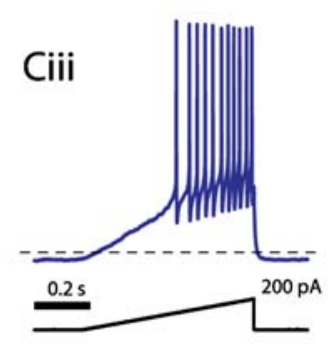

Diii

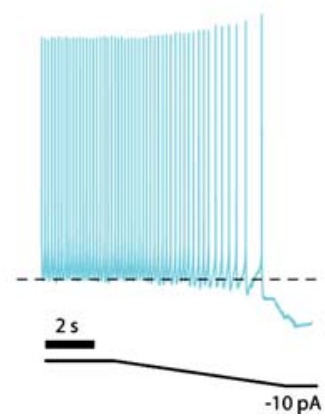

DIC even though easily distinguished in fluorescence imaging. $C$ Example recording from an inherently silent GlyT2+ cell. $\mathrm{C} i$ In cellattached mode only occasional action potentials are seen. Cii After breaking into whole-cell configuration with no bias current $\left(I_{\mathrm{cmd}}=\right.$ $0 \mathrm{pA}$ ), the membrane potential of the cell remains below spike threshold until depolarized with current injection (Ciii). In Cii and Ciii, the bottom panel depicts the bias (command) current $\left(I_{\mathrm{cmd}}\right)$; dashed line in the upper panel marks $-50 \mathrm{mV}$. Traces in $C i-C i i i$ are from the same cell. Note the different time scale in Ciii. D Example recording from spontaneously active GlyT2+ cells. $D i$ In cell-attached mode, the cell fires spontaneously. Dii After breaking into whole-cell mode with no bias current $\left(I_{\mathrm{cmd}}=0 \mathrm{pA}\right)$, spontaneous firing continues. Diii When a small amount of hyperpolarizing current (in this example, $-10 \mathrm{pA})$ is injected into the cell, the firing frequency slows down and ultimately ceases. Traces in $D i$ and Dii are from a single cell, Diii is from a different one. Note the different time scale in Diii

depolarizing current injection (Fig. 1 (Ciii)). The other presumed glycinergic neurons (21 out of 38 ) fired spontaneously both in external recording (at $8.3 \pm 0.7 \mathrm{~Hz}$; Fig. 1 (Di)) and in whole-cell current clamp with no bias current (at $10.4 \pm 1.4 \mathrm{~Hz}$; Fig. 1 (Dii)) and only ceased firing when they were hyperpolarized by negative current injection

established, the resting membrane potential (RMP) in these inactive GlyT2+ cells ("Gly-I") quickly stabilized below the action potential (AP) threshold (RMP $=-58.1 \pm 1.8 \mathrm{mV}$ (liquid junction potential (LJP) corrected value, $-72.6 \mathrm{mV}$ ), $n=12$; Fig. 1 (Cii)) with no injected bias current. In all of such cells, however, action potentials could be evoked by 
(Fig. 1 (Diii)). We termed these the active GlyT2+ ("GlyA") cells.

In addition to spontaneous firing behavior, the AP waveform has proved to be a useful feature for distinguishing the various DCN cell types [6] and comparison of AP waveforms in Gly-I and Gly-A cells revealed additional differences between these cells (Fig. 2 (A and B)). As is evident from the averaged action potential shapes (Fig. 2 (A); $n=16$ Gly-A and 11 Gly-I cells; for details of how the average waveforms and statistics were obtained, see Methods), the Gly-A cells displayed slow, deep AHPs reminiscent of that previously shown as a characterizing feature of the GAD+ cells [6] whereas the Gly-I cells had a fast and often biphasic AHP similar to GAD- cells. This difference was quantified by comparison of the peak $V_{\mathrm{m}}$ of the AHPs as well as the time-to-AHP peak relative to the AP peak (most negative $V_{\mathrm{m}}$ in AHP (AHP peak), Gly-A, $-53.3 \pm 2.6 \mathrm{mV}$ (LJP corrected value, $-67.8 \mathrm{mV}$ ); Gly-I, $-43.7 \pm 4.1 \mathrm{mV}$ (LJP corrected value, $-58.2 \mathrm{mV}$ ); $p<0.05$; AHP peak time, Gly-A, $17.0 \pm 2.5 \mathrm{~ms}$; Gly-I, $3.7 \pm 1.5 \mathrm{~ms}$; $p<0.001)$. Also, the Gly-A cells had broader APs than the
Gly-I as is evident when the average AP traces are examined in more detail (Fig. 2 (Aii and Bi), AP halfwidth (HW): Gly-A, $1.54 \pm 0.11 \mathrm{~ms}$; Gly-I: $0.82 \pm 0.06 \mathrm{~ms}$; $p<0.001)$. Even though the ranges of AP HW value ranges in Gly-I and Gly-A cells overlapped slightly (as seen in Fig. 2 (Bi)), plotting the AP HWs against estimated cell capacitance values $\left(C_{\mathrm{m}}\right.$; Fig $2(\mathrm{Ci})$; see Methods) revealed that the Gly-A cells firing broad APs clustered around $40 \mathrm{pF}$, whereas the Gly-I cells with fast APs had significantly larger capacitances (Gly-A, $41.5 \pm 2.3 \mathrm{pF}, n=$ 15; Gly-I, $103.7 \pm 17.4$ pF, $n=10$; $p<0.001$; Fig. 2 (Cii)). These results indicate the presence of two types of presumed glycinergic neurons in the DCN: (1) spontaneously active, smaller Gly-A cells with broad action potentials and slow monophasic AHPs and (2) silent, larger Gly-I cells with narrower APs and fast biphasic AHPs.

\section{The Dynamic Behavior of Gly-I and Gly-A Cells}

The dynamics of AP firing during injection of depolarizing current pulses are commonly used for functional classifica-
Fig. 2. Action potential $(A P)$ waveforms in Gly-A (light blue) and Gly-I (dark blue) neurons. Ai Peak-aligned grand averages of APs. Width of the trace denotes SEM values. The same traces are shown with an expanded time scale in Aii. Note the faster AHP (Ai; arrow) and shorter AP width (Aii) of Gly-I cells as compared with Gly-A cells. $B$ Comparison of key metrics of the AP waveform. $B i$ AP half-width in ms; Bii AP threshold, AP peak, and AHP peak voltages in millivolts; Biii AHP peak time in ms. Vertical lines denote SEM values; circles denote individual values. $C$ AP half-width (AP HW) and cell capacitance $\left(C_{\mathrm{m}}\right)$ define cell populations. $C i$ plot of AP halfwidth values against $C_{\mathrm{m}}$ shows that the Gly-A (light blue) and Gly-I (dark blue) cell populations are almost entirely separate. $C i i C_{\mathrm{m}}$ distribution in Gly-A and Gly-I cells. Asterisks denoting statistical significance, ${ }^{*} p<0.05 ; * * * p<0.001$

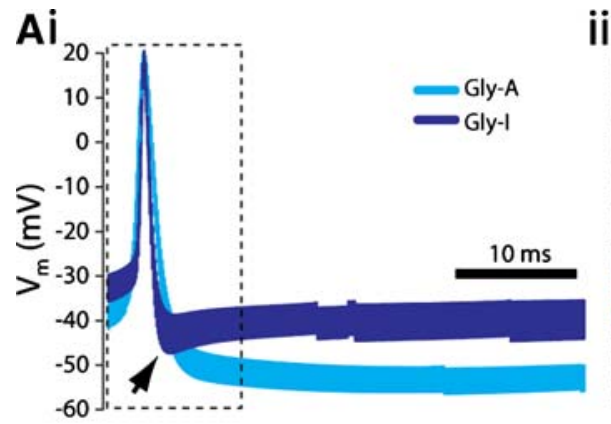

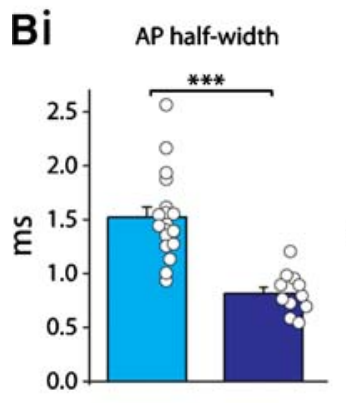

Ci

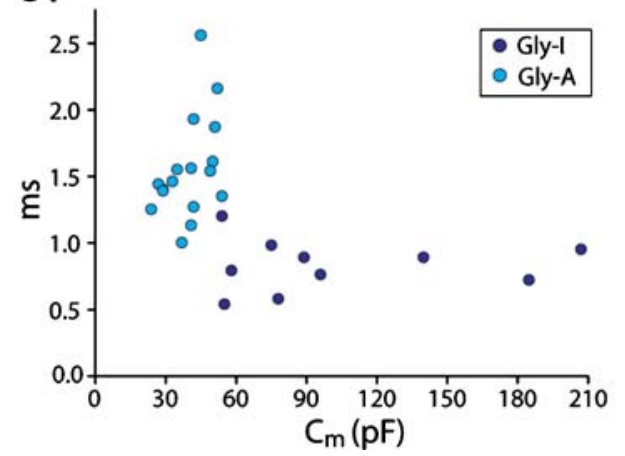

ii

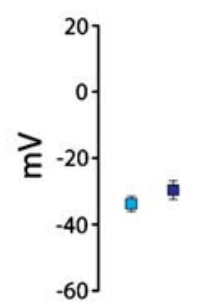

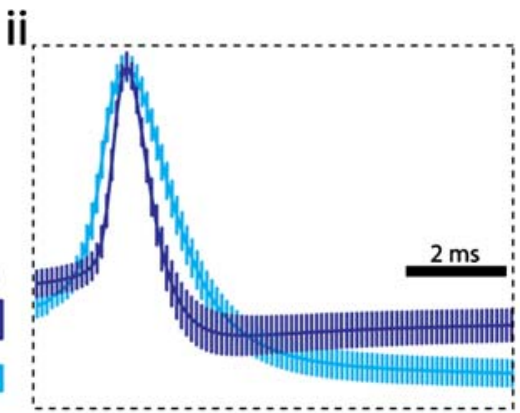

百

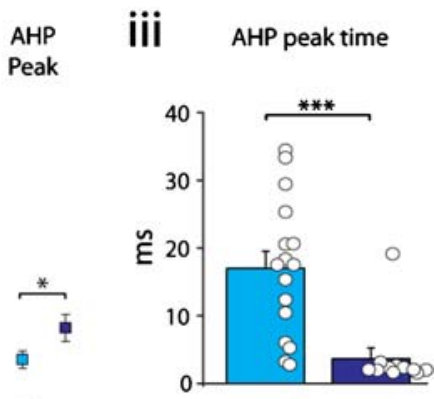

ii

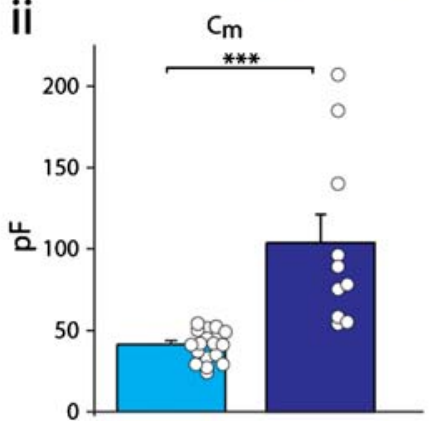


tion of neuronal phenotypes, and differences in firing frequency versus injected current $(f-I)$ may reflect variation in the ion channel makeup between cells. Furthermore, these measures provide information related to the sensitivity of the neuron to synaptic input and the capability of the neuron to respond and relay tonic and phasic inputs, respectively. AP firing dynamics of the glycinergic cells was studied using sets of depolarizing current steps, normalized on the cell $C_{\mathrm{m}}$ (see Methods) so that results obtained in cells of varying sizes could be directly compared (Fig. 3). Prior to the step depolarization the spontaneously active Gly-A cells were hyperpolarized

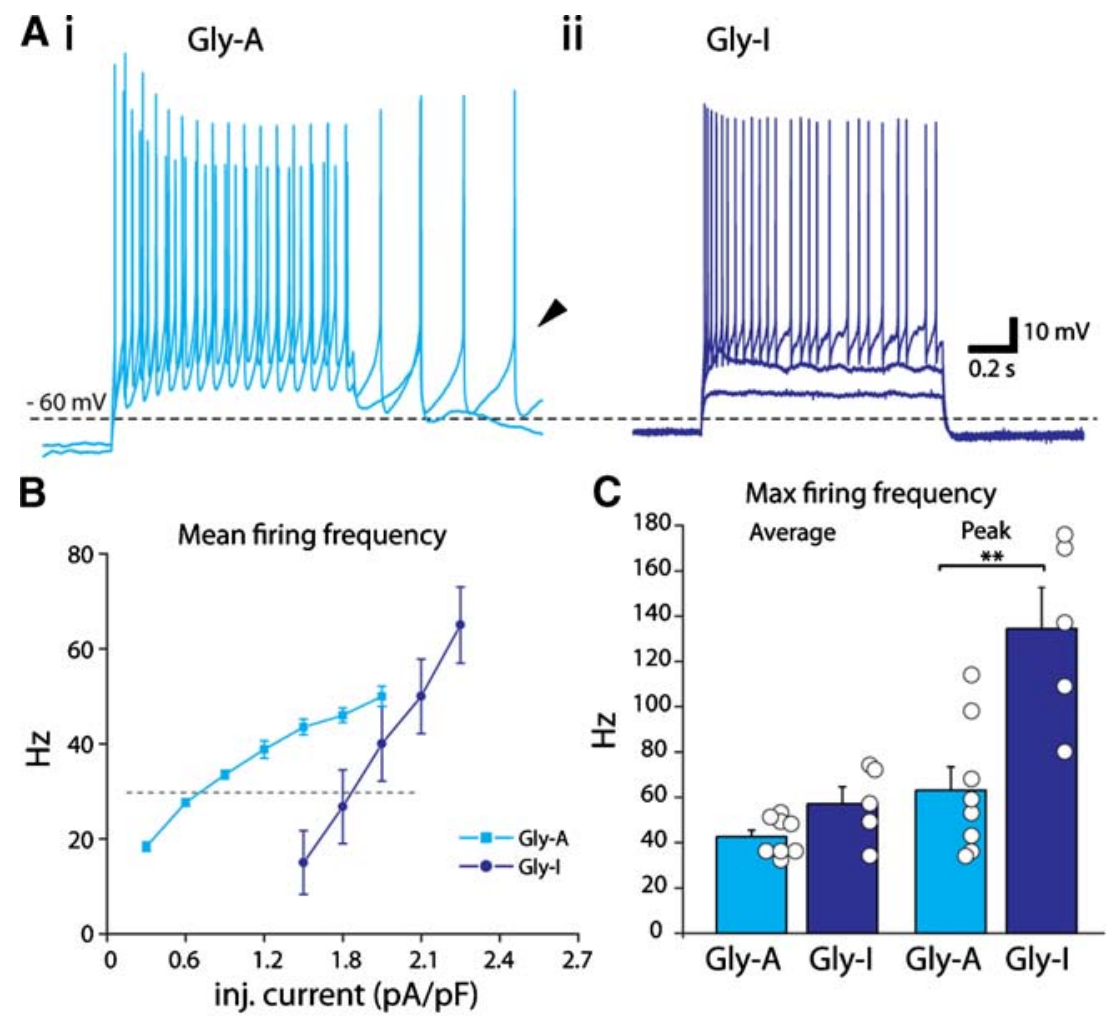

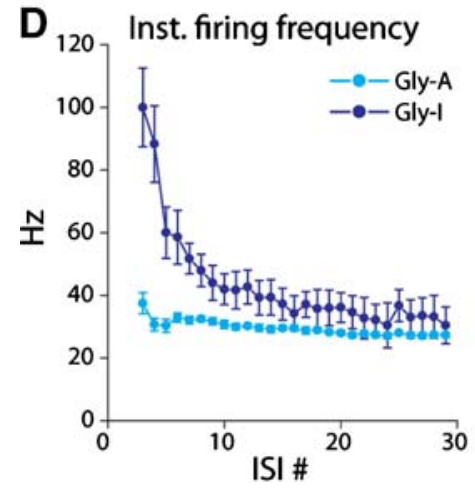

Fig. 3. Action potential firing dynamics. $A$ Example traces of firing dynamics of Gly-A (i) and Gly-I (ii) neurons. Voltage responses evoked by 1 -s long 0.6 and $0.9 \mathrm{pA} / \mathrm{pF}(i)$ and $0.9,1.5$, and $2.4 \mathrm{pA} / \mathrm{pF}$ (ii) current steps from subthreshold membrane potentials. Note that the Gly-A cell continued to fire action potentials after offset of the depolarizing pulse (arrowhead in $A i$ ). B Plot of mean evoked firing frequency vs. the injected current (I-f) of Gly-A and Gly-I cells. Note that the Gly-I cells require larger depolarizing current injections to start firing than the Gly-A cells but reach higher frequencies. $C$ Comparison of maximal average (left) and peak (right) frequencies reached by Gly-A and Gly-I cells. Note the significantly higher peak firing frequencies evoked in Gly-I cells. Vertical lines denote SEM values; circles denote values obtained from individual cells. Asterisks

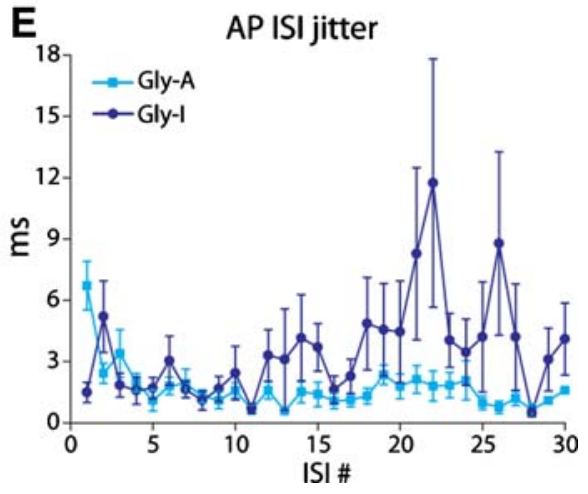

denote statistical significance, ${ }^{* *} p<0.01$. $D$ Comparison of instantaneous firing during depolarizing step reveals stronger frequency attenuation in Gly-I cells than in Gly-A cells. The values are obtained from trains of APs evoked by 1-s long depolarizations during which the average firing frequency was closest to $30 \mathrm{~Hz}$ (marked with dashed horizontal line in Fig. 4b). Vertical bars denote SEM values. $E$ Comparison of firing regularity in terms of absolute magnitude of inter-spike interval (ISI) jitter during depolarizing current steps that evoke $\sim 30 \mathrm{~Hz}$ firing. After the initial slowdown of firing in Gly-A cells (light blue), the firing becomes stable and regular (with not more than 1-2 ms of jitter). In the Gly-I cells firing becomes less irregular during a train, with up to $20 \mathrm{~ms}$ of difference between two consecutive ISIs. In $D$ and $E$, ISI\# counts the inter-spike intervals during the step 
using a small bias current (up to $-50 \mathrm{pA}$ ) to a stable subthreshold level ( -60 to $-70 \mathrm{mV}$; LJP corrected, -75 to $-85 \mathrm{mV}$ ) as was done in the earlier study [6]; the inactive Gly-I cells were allowed to stay at their RMP without injection of bias current. Representative examples of responses to depolarizing current steps in Gly-I and Gly-A cells are shown in Fig. 3 (A), and the results are summarized in Fig. 3 (B) ( $\mathrm{f}-\mathrm{I}$ relationship) and $\mathrm{C}$ (adaptation of spike rate).

Similarly to the previously described DCN cell types the Gly-A cells held at subthreshold potentials could be made to fire repetitively by small depolarizations (the smallest one used was $0.3 \mathrm{pA} / \mathrm{pF}$, which for an average-sized cell $(40 \mathrm{pF})$ corresponded to $12 \mathrm{pA}$; Fig. $3(\mathrm{Ai})$ ) and the mean firing frequency during the step increased sublinearly with larger injected currents until saturating around $50 \mathrm{~Hz}$ (Fig. 3 (B)). Attempts at making the cells fire faster than $50 \mathrm{~Hz}$ using larger current steps resulted in the cells reaching a depolarized plateau state characterized by radical decrease of AP amplitude as well as disruption of firing ("depolarization block"; [17]).

In contrast to the Gly-A cells and all other DCN cell types that we described, the Gly-I cells usually required larger steps of depolarization to reach firing threshold (typical minimal step was $1.5 \mathrm{pA} / \mathrm{pF}$; Fig. 3 (B)). Note that this was not due to the use of $C_{\mathrm{m}}$-normalized current amplitudes, as the steps induced membrane depolarizations that were comparable to those seen using similar sized steps in Gly-A cells (compare traces in Fig. 3 (Ai and ii), where the current step sizes are $0.9,1.5$, and $2.4 \mathrm{pA} / \mathrm{pF}$ in the GlyI and 0.6 and $0.9 \mathrm{pA} / \mathrm{pF}$ in the Gly-A cell). The Gly-I cells could reach slightly (but not significantly) faster average frequencies during the step depolarizations than the Gly-A cells (Gly-I, $57.2 \pm 7.4 \mathrm{~Hz}, n=5$; Gly-A, $42.6 \pm 3.0 \mathrm{~Hz}, n=8$; $p=0.058$; Fig. 3 (C), left). The faster average frequencies reached by Gly-I cells were mainly due to their ability to fire a higher-frequency burst of APs at the start of the depolarization (maximum instantaneous frequency during a step depolarization in Gly-I cells, $134.1 \pm 18.1 \mathrm{~Hz}, n=5$; Gly-A, $61.0 \pm 9.3 \mathrm{~Hz}, n=9 ; p<0.01$; Fig. 3 (C), right). However, the firing frequency in Gly-I cells declined quickly during a depolarizing current step and sometimes the cells stopped firing altogether. Figure 3 (D) shows instantaneous firing frequencies for series of APs during current steps where the mean firing frequency was closest to $30 \mathrm{~Hz}$. Conversely, the Gly-A cells showed less accommodation during a depolarizing step and often fired a few additional APs after the offset of the depolarization (see arrowheads in Fig. 3 (Ai)) whereas firing in Gly-I cells always stopped immediately when the depolarizing step ended. In addition, the Gly-A cells behaved similar to the other, previously described DCN cell types in that their firing was regular, whereas action potentials of Gly-I cells were irregularly spaced even during steady-state continuous firing. Figure 3 (E) shows the development of ISI jitter during steps where the cells fired on average at $\sim 30 \mathrm{~Hz}$ (see Methods). After the initial attenuation of firing frequency the Gly-A cells fire with very little jitter in the spike intervals (average absolute jitter during spike intervals 10 $301.39 \pm 0.22 \mathrm{~ms}, n=8$ cells). In the Gly-I cells variability in ISI stays higher even after the firing frequency has stabilized $(4.17 \pm 2.63 \mathrm{~ms}, n=5$ cells; $p<0.001)$.

\section{Gly-A Cells Project Locally While Gly-I Cells Project} Toward the Cerebellar Cortex

Seven Gly-I and ten Gly-A cells were successfully filled and stained with biocytin; examples of both cell types are shown in Fig. $4 \mathrm{a}$ and the main morphometric parameters are summarized in Fig. 4b. As the Gly-A cells exhibited a smaller membrane capacitance than the Gly-I cells (Fig. 2 (Cii)), we were not surprised to find that they also had smaller cell bodies and shorter dendritic trees with fewer branches than the Gly-I cells (soma length: Gly-A, $16.1 \pm 1.3 \mu \mathrm{m}$; GlyI, $23.3 \pm 1.4 \mu \mathrm{m} ; p<0.005$; soma area: Gly-A, $130 \pm 13 \mu \mathrm{m}^{2}$; Gly-I, $256 \pm 22 \mu \mathrm{m}^{2} ; p<0.001$; extension of the dendritic tree: Gly-A, $140 \pm 16 \mu \mathrm{m}$; Gly-I, $199 \pm 15 \mu \mathrm{m} ; p<0.05 ; n$ of primary dendrites: Gly-A, 2.9 \pm 0.3 ; Gly-I, 3.6 $\pm 0.2 ; p=$ 0.069 ; BI: Gly-A, $1.14 \pm 0.09$; Gly-I, $1.7 \pm 0.2 ; p<0.05$; as for how these measurements were made, see Methods).

What was surprising, however, was that all of the seven Gly-I cells that we could recover for biocytin stain and morphological analysis sent a long-distance axon projecting towards the dorsal or lateral aspects of the DCN; five of these projections were found to extend out of the dorsolateral DCN border and into the white matter. Furthermore, in three of these cases the axons could be traced up to where they entered the granule cell layer (GCL) within which they could be followed for several hundreds of micrometers (an example of the latter is presented in Fig. 4c). Such projecting axons were not seen in any of the ten stained Gly-A cells or in the GAD+ or GADnS cells studied in our previous work $(n=21$ and 16 , respectively [6]; note that the previous study did not show strong support for cell morphology as a distinguishing feature between $\mathrm{GAD}+$ and $\mathrm{GAD}-$ cells). Instead, the axons of the Gly-A cells were confined to areas near the cell body (arrowheads in Fig. 4a). The Gly-I cells that seemed to project to the cerebellar cortex often also displayed axon collaterals that branched and terminated in areas close to their cell body (arrowheads in Fig. 4c).

\section{Comparison of GlyT2+ Cells with Other DCN Cell Types}

It has been repeatedly shown that at least some of the glycinergic cells in DCN colocalize GABA or GAD67 and 
A
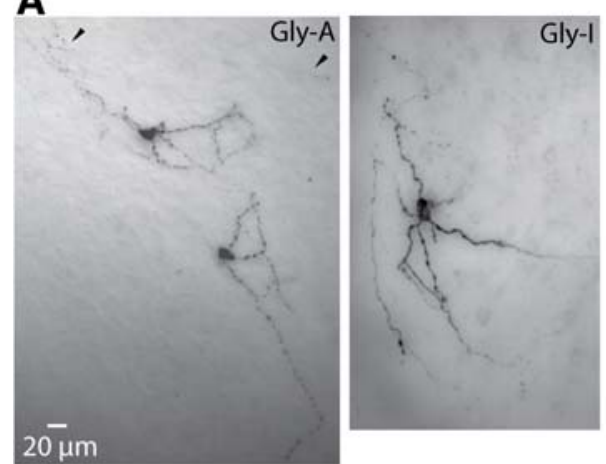

C

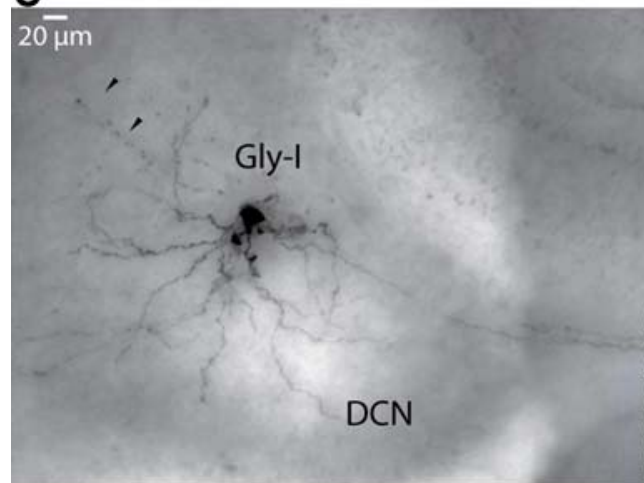

Fig. 4. Morphology of biocytin-filled GlyT2+ DCN neurons. A $Z$ stack projections of two spontaneously active (Gly-A; left) and one inactive (Gly-I; right) GlyT2+ cell. The arrowheads point to axon ramification near a Gly-A cell body. Scale bar for both images, $20 \mu \mathrm{m}$. B Quantitative morphometry of Gly-A (light blue) and Gly-I (dark blue) cells: left soma area (in $\mu \mathrm{m}^{2}$ ) and maximal extension of resolvable dendrites (in $\mu \mathrm{m}$ ); right number of resolvable primary dendrites and branch index value. The vertical lines denote SEM values and white circles mark the individual values. Asterisks denoting

that some but not all of the GABAergic cells in DCN are glycinergic $[1,9,18]$. Therefore, the question that needed addressing was the relationship of the GlyT2+ cells to the previously described GAD + cells and whether either of the presently described GlyT2+ cell groups would be a subpopulation of the GAD+ or the GADnS cells.

It seems unlikely that the intrinsically silent Gly-I cells would correspond to either GADnS or GAD+ cells, as all of the cells belonging to those groups that have been reliably recorded in our laboratory so far (during works reported in [6] and [19]) show a consistent and strong tendency to spontaneous regular firing. Also none of the $25 \mathrm{GAD}+$ or 21 GADnS cells successfully stained during this or the previous studies in our laboratory $[6,19]$ showed any indication of an axon projecting far from their own dendritic fields.

It could be argued, however, that the inactivity of some cells in the present work could be due to the more mature
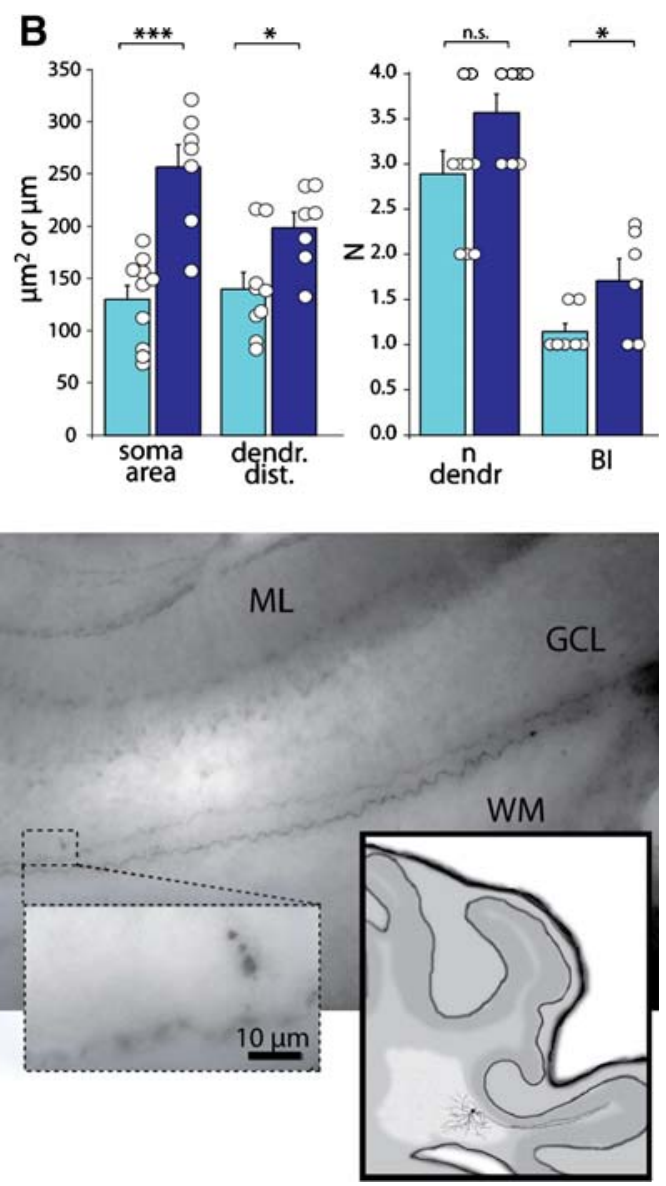

statistical significance: $* * * p<0.001 ; * p<0.05$; n.s. $p>0.05$. C A collage of $Z$ stack projections from a Gly-I cell that projected outside the DCN, into the cerebellar granule layer. The right inset shows a drawing of the location of the cell and its axon; the left inset shows a magnification of the area where a small axonal branch is seen within the granule cell layer. Arrowheads to the left side of the cell body mark axon collateral terminals within the DCN. $M L$ molecular layer, $G C L$ granule cell layer, $W M$ white matter

state of the cells or of the synaptic circuitry at P20-28 when compared with the previous studies performed in younger (P14-19) animals. To investigate this possibility and to compare the behavior of the inactive GlyT2+ (Gly-I) and active GlyT2+ (Gly-A) cells to those of other cell types in this age range, we also recorded six GADnS and 18 GAD+ cells in P20-24 GAD67/GFP+ mice. All of such cells recorded were spontaneously active, both in cell-attached $(\mathrm{GAD}+, 10.7 \pm 0.7 \mathrm{~Hz}$; GADnS, $8.4 \pm 1.1 \mathrm{~Hz} ; \mathrm{GAD}+$ and GADnS cell example traces are shown in Fig. 5 (Ai)) and in whole-cell current clamp with no bias current $(\mathrm{GAD}+, 11.0 \pm$ $1.5 \mathrm{~Hz}$; GADnS, $10.3 \pm 2.4 \mathrm{~Hz}$; Fig. 5 (Bi)). Additional experiments (data not shown) using the GADnS and GAD+ cells as well as six GADnL from P20-24 GAD67/GFP+ mice did not reveal qualitative differences in cell morphology, passive membrane properties, action potential waveform, frequency adaptation, or spontaneous postsynaptic 

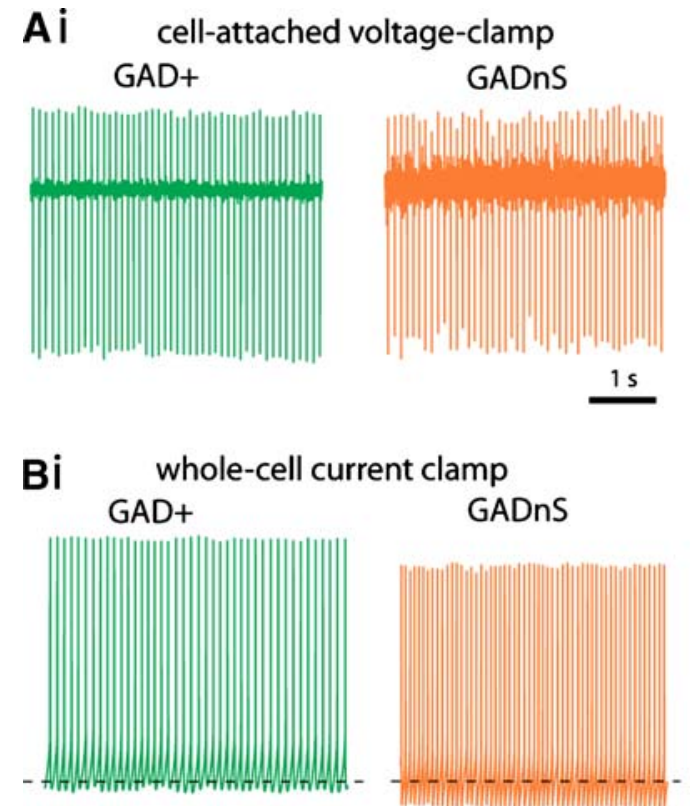

amp

GADnS

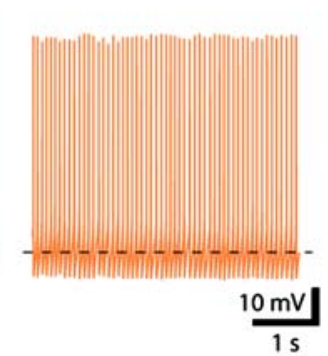

ii Firing frequency

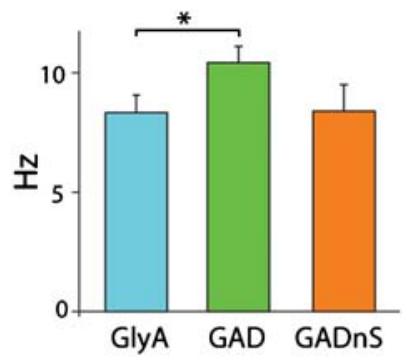

ii Firing frequency

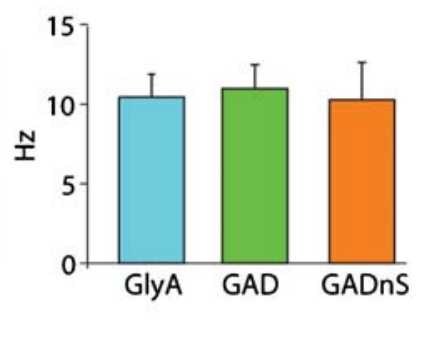

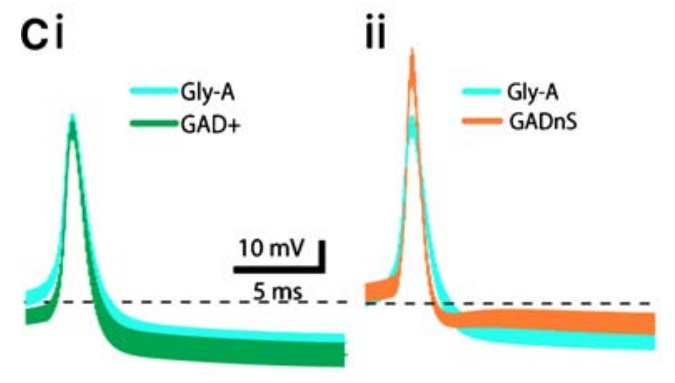
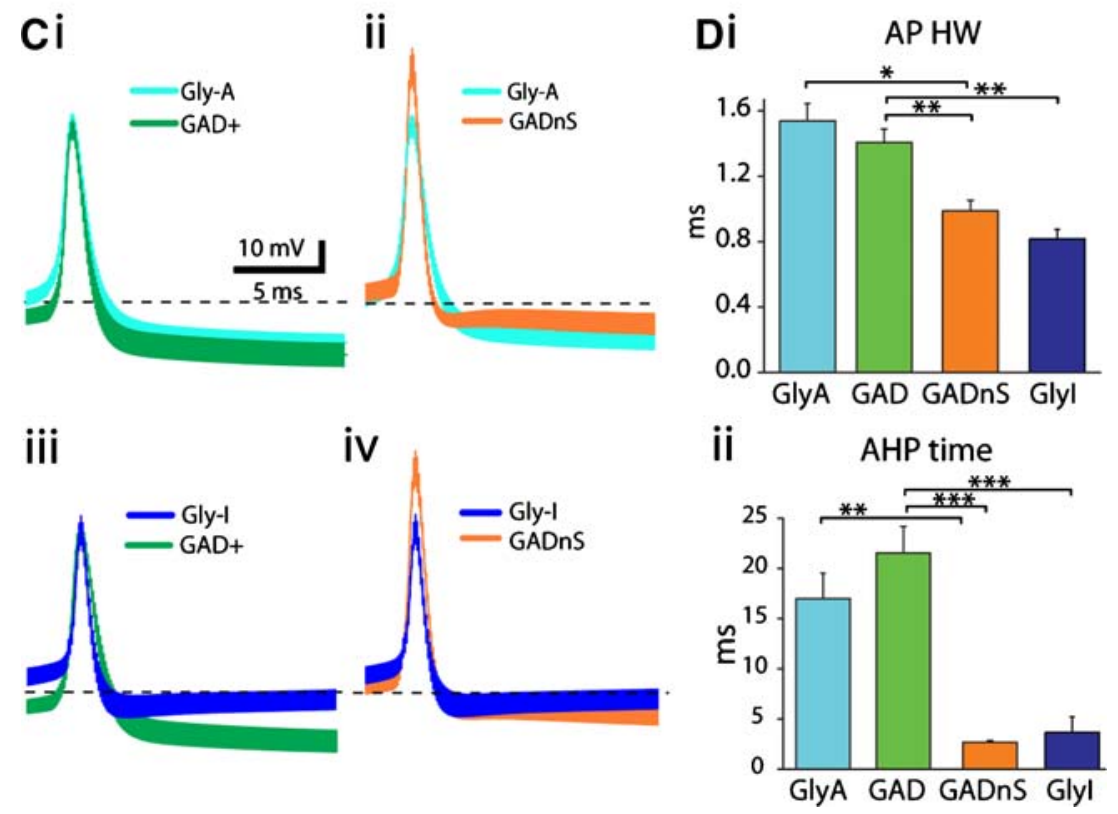

Fig. 5. Comparison of Gly-A (light blue) and Gly-I (dark blue) cells with GAD+ (green) and GADnS (orange) cells. A Spontaneous firing in cell-attached mode. $A i$ Examples of cell-attached voltage-clamp recordings from GAD+ (left) and GADnS (right). All of the GAD+ and GADnS cells were spontaneously active. Aii Comparison of average firing frequencies in Gly-A, GAD + , and GADnS cells. GAD+ cells were found to fire slightly faster than Gly-A and GADnS cells. $B$ Spontaneous firing in whole-cell current clamp with no bias current injection. Dashed line denotes $-40 \mathrm{mV}$. Bi Examples of whole-cell current clamp recordings from GAD+ (left) and GADnS (right) cells. Bii Comparison of average firing frequencies in Gly-A, GAD+, and GADnS cells. No significant differences were found. $C$ Comparison of peak-aligned grand average action potential waveforms between Gly-
A and GAD + $(\mathrm{Ci})$, Gly-A and GADnS (Cii), Gly-I and GAD+ (Ciii), and Gly-I and GADnS (Civ) cell types. Width of the trace denotes SEM. Dashed line denotes $-40 \mathrm{mV}$. D Bar graphs comparing the GlyA, Gly-I, GAD+, and GADnS cells in terms of the two key AP shape parameters, AP half-width $(D i)$ and AHP peak time (Dii). In both measures GlyA and GAD+ cells as well as Gly-I and GADnS cells were virtually indistinguishable. On the other hand, Gly-A and GADnS cells as well as Gly-I and GAD + cells were found to be highly dissimilar. Asterisks denoting statistical significance: $* * * p<0.001$; ${ }^{* *} p<0.01 ; * p<0.05$. For graphical clarity, statistical significance comparisons between values obtained from Gly-A and Gly-I cells are not shown in this figure. Gly-I cells were not spontaneously active and are thus omitted in $A$ and $B$ 
current properties between the juvenile (P14-20; $[6,19])$ and young adult (P20-24) animals. These results thus support the earlier assumptions that most of the DCN neurons are electrophysiologically mature by P14.

Based on spontaneous firing frequency, cell body size and $C_{\mathrm{m}}$, the Gly-A cells overlap with the GAD+ and GADnS cells described earlier [6]. The main distinguishing feature between GAD + and GADnS cells was, however, their AP waveform and especially the presence or absence of a fast AHP (fAHP). We therefore compared the population averages of AP shapes in Gly-A cells to those obtained from GAD+ and GADnS cells in P20-24 GAD67/ $\mathrm{GFP}+$ mice, recorded under similar conditions (room temperature, no bias current; $n=13 \mathrm{GAD}+$ and five GADnS cells, respectively; Fig. 5 (C and D)). The Gly-A cells were characterized by broad APs and slow, simple AHPs with little evidence for a fAHP component (Fig. 2 (A); AHP peak time comparison with Gly-I cells in Fig. 2 (Biii)). Unsurprisingly, a comparison of the cell groups demonstrated that the AP waveforms in Gly-A cell groups were similar to the GAD+ cells (Fig. $5(\mathrm{Ci})$ ) and clearly different from GADnS cells (Fig. 5 (Cii)) in terms of AP half-width and time to first AHP minimum as well as other parameters (AP HW: GAD+, $1.4 \pm 0.08 \mathrm{~ms}$; GADnS, $0.99 \pm 0.06 \mathrm{~ms} ; t$ test for GAD + vs. Gly-A, $p=0.35$; GAD + vs. Gly-I, $p<$ 0.001 ; GADnS vs. Gly-A, $p<0.05$; ANOVA over all groups: $F=8.74, p<0.001$; AHP peak time: $\mathrm{GAD}+, 21.5 \pm$ $2.6 \mathrm{~ms}$; GADnS, $2.7 \pm 0.2 \mathrm{~ms} ; t$ test for GAD+ vs. Gly-A, $p=0.23$; GAD + vs. Gly-I, $p<0.001$; GADnS vs. Gly-A, $p<0.01$; ANOVA over all groups: $F=18.8, p<0.001$; Fig. 5 (Di and ii); other parameters for GAD+ and GADnS along with Gly-A and Gly-I cells are given in Table 1). These similarities suggest that the Gly-A cells are in fact members of the GAD+ cell group, which would correspond to the GABA and glycine co-expressing cell group described earlier [1, 7-9].

The faster AP duration and fAHP peak in Gly-I cells differed clearly from GAD + cells and resembled those seen in GADnS cells both in P20-24 (compare average AP waveforms in Fig. 5 (Ciii and iv); $t$ test for Gly-I vs. GADnS for AP HW: $p=0.098$; AHP peak time: $p=0.68$; Fig. 5 (D) and Table 1) and P14-20 animals (compare values with those given in [6]). Gly-I cells were, however, clearly distinct from GADnS cells because only the former exhibit a subthreshold resting membrane potential as well as irregular firing pattern. Therefore, we have no reason to believe that the inactive Gly-I cells would simply be more mature GADnS cells.

\section{Discussion}

\section{Glycinergic Neurons of the DCN}

Traditionally, the neurons in DCN have been categorized into large or small cells, with the large cells being glutamatergic projection neurons (likely corresponding to the GADnL cells; [6]), and the small cells comprising of various local neurons (GADnS and GAD+ cells; [6]) as well as the GABAergic neurons controlling the activity of the inferior olive (IO) [20]. It is known that a large portion of the GABAergic cells co-express glycine while there are also non-GABAergic glycinergic and non-glycinergic GABAergic cells [1,7-9]. The GABAergic/non-glycinergic

Table 1 Electrophysiological parameters of the cell types examined

\begin{tabular}{|c|c|c|c|c|c|c|c|c|c|c|c|c|}
\hline & \multicolumn{3}{|l|}{ Gly-A } & \multicolumn{3}{|l|}{ Gly-I } & \multicolumn{3}{|l|}{ GAD+ } & \multicolumn{3}{|l|}{ GADnS } \\
\hline & Mean & SEM & $N$ & Mean & SEM & $N$ & Mean & SEM & $N$ & Mean & SEM & $N$ \\
\hline$C_{\mathrm{m}}(\mathrm{pF})$ & 41.5 & 2.3 & 15 & 103.7 & 17.4 & 10 & 50.5 & 3.8 & 13 & 77.0 & 9.1 & 6 \\
\hline AP threshold (mV) & -32.7 & 2.5 & 16 & -29.6 & 2.8 & 11 & -39.1 & 1.6 & 13 & -34.0 & 3.1 & 5 \\
\hline AP peak $V_{\mathrm{m}}(\mathrm{mV})$ & 17.1 & 3.1 & 16 & 17.4 & 2.9 & 11 & 15.5 & 2.2 & 13 & 32.3 & 5.5 & 5 \\
\hline AP half-width (ms) & 1.54 & 0.11 & 16 & 0.82 & 0.06 & 11 & 1.4 & 0.08 & 13 & 0.99 & 0.06 & 5 \\
\hline AHP peak time (ms) & 17.0 & 2.5 & 16 & 3.7 & 1.5 & 11 & 21.5 & 2.6 & 13 & 2.7 & 0.2 & 5 \\
\hline AHP peak $V_{\mathrm{m}}$, absolute $(\mathrm{mV})$ & -53.3 & 2.6 & 16 & -43.7 & 4.1 & 11 & -54 & 2.0 & 13 & -45.9 & 2.0 & 5 \\
\hline AHP peak $V_{\mathrm{m}}$, relative to AP threshold $(\mathrm{mV})$ & -20.5 & 1.0 & 16 & -14.1 & 1.8 & 11 & -14.9 & 1.7 & 13 & -12 & 2.2 & 5 \\
\hline Firing frequency, cell attached & 8.33 & 0.73 & 22 & NA & NA & NA & 10.4 & 0.68 & 17 & 8.4 & 1.1 & 4 \\
\hline Firing frequency, whole cell, no bias current & 10.4 & 1.4 & 14 & NA & NA & NA & 11.0 & 1.5 & 12 & 10.3 & 2.4 & 6 \\
\hline Max. average firing during depolarizing steps & 42.6 & 3.0 & 8 & 57.2 & 7.4 & 5 & 36.6 & 4.0 & 5 & 59.0 & 6.1 & 4 \\
\hline $\begin{array}{l}\text { Max. peak frequency during depolarizing } \\
\text { steps }\end{array}$ & 63.1 & 10.3 & 8 & 134.4 & 18.2 & 5 & 72.7 & 11.8 & 5 & 126.8 & 19.1 & 4 \\
\hline
\end{tabular}

Note that the values are not corrected for the liquid junction potential (calculated value, $14.5 \mathrm{mV}$ )

$S E M$ standard error of the mean, $C_{m}$ membrane capacitance, $N A$ value not available 
cells probably include the small IO-projecting GABAergic cells because no glycinergic terminals have been found in the rodent IO [10].

We found two types of GFP-expressing neurons in the DCN ("Gly-A" and "Gly-I") in the GlyT2-eGFP mice, both of which can be presumed to be glycinergic in nature as the GlyT2 expression has been tightly linked to glycinergic neurons in the adult DCN $[2,12]$. A definite proof of this presumption will require paired recordings from pre- and postsynaptic cells and demonstration of glycinergic synaptic potentials, experiments that will unfortunately be technically difficult in slices, especially for the putative nucleocortical synaptic connections, given that all of the long-range GlyT2+ axons that we stained were cut during slice preparation.

The electrophysiological characteristics of the Gly-A cells differed clearly from the GADnS cells described in [6], but overlapped with the GABAergic cell population described in the same study ("GAD+") suggesting that the Gly-A cells co-express GABA. In contrast, the Gly-I cells likely represent non-GABAergic glycinergic neurons as their action potential shape parameters were clearly distinct from GAD + cells and because they, unlike all other DCN neurons, did not fire spontaneously. Finding silent neuronal population in a structure where all neurons described earlier are spontaneously active was surprising. In addition to the sparseness of the Gly-I cells, their absence in earlier recordings may be due to the fact that the glycinergic circuitry is known to mature relatively late in the DCN [5] while the majority of slice recordings by us or other laboratories so far have been obtained from younger animals.

The most unexpected finding, however, was that all of the successfully stained Gly-I cells had an axon projecting outside the vicinity of their dendritic trees and in several cases the axon could be followed to the cerebellar cortex. The Gly-A cell axons were never seen to project far from the cell body, and also were characterized by smaller and less complex dendritic trees, smaller $C_{\mathrm{m}}$ and cell body size (Figs. 2 (C) and 4b). When both $C_{\mathrm{m}}$ and AP HW are taken into account, the two populations are almost entirely nonoverlapping (Fig. 2 (Ci)). Interestingly, Bagnall and colleagues also recently found two populations of glycinergic neurons in the fastigial nucleus that are clearly separable by similar morphological and electrophysiological features [21].

The Gly-A cells and the GAD + cells recorded in GAD67/GFP+ mice were indistinguishable in almost all parameters examined (Fig. 5 and Table 1). The only features where we found a difference reaching statistical significance were firing frequency in cell-attached mode and cell capacitance, where the GAD + cells ranged slightly larger in terms of $C_{\mathrm{m}}$ than the Gly-A cells (GAD+ vs. Gly-
A, $p=0.048)$. Regardless of these two modest differences, our data strongly suggest that Gly-A cells are a subgroup of the GAD+ cells and thus correspond to the histologically defined group of GABA/glycine co-expressing neurons [1, 7-9]. This is in line with the earlier reports showing that the GABA/glycine co-expressing neurons are on average smaller in size [9].

As our previous work did not clarify the identity of the GADnS cells we initially wondered whether they might in fact be glycinergic rather than glutamatergic neurons. However, similarly to the GAD + cells, all the GADnS cells recorded in this and our previous study [6] fired spontaneously and we have seen no evidence for GADnS axons projecting outside DCN. Furthermore, even though the gross AP shape of Gly-I and GADnS cells was similar in terms of AP half-width and AHP, the cells in these two groups differed in several important ways. First, the AP firing in Gly-I cells was always highly irregular whereas the GADnS cells were regular firing (compare Fig. 3 (D and E) with Fig. 7 (B2) of [6]). Second, compared to the GADnS cells, the Gly-I cells required a higher threshold current (rheobase) for firing and the current-to-frequency relationship (f-I) did not saturate at $\sim 50 \mathrm{~Hz}$ (compare Fig. 3 (B) with Fig. 7 (B1) of [6]). Third, the Gly-I cells ranged larger in terms of $C_{\mathrm{m}}$ (this study) and cell body size (compare Fig. $4 \mathrm{~b}$ with the data presented in [6]). Therefore there is no strong support for our initial hypothesis that the GADnS cells would correspond to glycinergic neurons.

The molecular identity of the GADnS cells remains thus unverified, even though the simplest explanation for the fact that they are non-GABAergic [6] and the present results showing they are distinct from the GlyT2-expressing cell population would be that they are glutamatergic, putatively locally projecting neurons.

Thus, the current knowledge of the electrophysiology in various cell types in lateral DCN can be summarized as follows. Most of the lateral DCN neurons are spontaneous, regular pacemakers. The large glutamatergic projection neurons (GADnL) fire fast APs with clear fast AHP with little adaptation, whereas the GABAergic neurons (GAD+), at least part of which co-express glycine, fire broader APs with lower frequencies and some frequency adaptation. In contrast to these spontaneously firing pacemaker cell types, the Gly-I cells are intrinsically silent neurons that fire fast APs when depolarized but their firing is irregular. In addition to these three identified subgroups we have proposed that the small non-GABAergic, non-glycinergic neurons (GADnS) are a population that is separate from the GADnL cells. This is based on their electrophysiological features which are in between the GADnL and GAD+ cells: they fire faster spontaneous APs than the GAD + cells, display fast AHPs, but there is more frequency adaptation than in the GADnL cells. Notably, at this stage we do not 
know whether the IO-projecting neurons differ from the local GABAergic neurons in electrophysiological terms as they have not been specifically targeted in experiments. Furthermore, due to the known difficulties in obtaining reliable and stable whole-cell recordings in DCN slice preparations from animals older than P14 [21] we had to restrict the experiments to cool temperatures $\left(23-24^{\circ} \mathrm{C}\right)$ as we used older animals than previously in order to ascertain maturity of the glycinergic network in DCN [5]. In our previous work [6] we have shown that the temperature dependency of AP waveform parameters in other DCN neuron types can be described by Q10 value of $1.86 \pm 0.05$ (range 1.73-2.07), and that the firing behavior did not change qualitatively. Thus, we do not believe that the specific features of Gly-I cells described here would be simply an artifact of the recording temperatures, even though in vivo recordings of these neurons might reveal different spiking patterns due to presence of intact synaptic connections.

\section{Evidence for an Inhibitory Nucleocortical Projection}

As discussed above, an intriguing difference between the Gly-A and Gly-I cells was the presence of an axon projecting towards the cerebellar cortex in all of the seven successfully stained Gly-I cells (Fig. 4c). The projecting axons of Gly-I cells were directed towards the dorsolateral borders of the DCN and in several cases were seen to leave the DCN and enter the granule cell layer in the lateral cerebellar cortex. Such an axonal alignment towards the dorsolateral aspect of the DCN has been shown to be a defining feature of the nucleocortical projections [7], and even though we often saw axon collaterals of the Gly-I cells ramifying within the DCN, they never projected towards the vental edges of the DCN which would implicate projection to extracerebellar structures via the cerebellar peduncles. Furthermore, it has been recently found [21] that at least in juvenile (P10-14) mice there are no glycinergic neurons projecting from the lateral DCN to extracerebellar structures. Nevertheless, as the glycinergic circuitry in DCN is not expected to be mature by $\mathrm{P} 14$, it is possible that a glycinergic projection from DCN to areas outside the cerebellum would develop later than what was studied in [21].

Earlier studies using tracing and electrophysiological methods have demonstrated a topographically conserved nucleocortical projection [7, 22-26]. It has been assumed that this projection forms part of the excitatory mossy fibers as only few or no GABAergic neurons have been found among those projecting to the cerebellar cortex [7, 23, 24, $28]$. However, to our knowledge the tracing of nucleocortical neurons has not been combined with glycine or GlyT2 immunohistochemistry. Therefore, the possibility remains that the nucleocortical projection could include glycinergic axons accompanying the collaterals of other projecting DCN neurons [21]. In addition, evidence for nucleocortical neurons that express neither GABA nor glutamate [25] supports the hypothesis that the nucleocortical Gly-I cells are indeed non-GABAergic and therefore purely glycinergic, inhibitory neurons.

\section{Significance in the Cerebellar Circuitry}

The localization of glycinergic synapses within the DCN has been studied intensively $[1,5,27]$ where they are mainly found on cell bodies of non-GABAergic, nonglycinergic and thereby presumably glutamatergic neurons [27]. The density of these synapses is not high and they probably correspond to glycinergic terminals of only one or few presynaptic fibers, in contrast with the extremely heavy GABAergic Purkinje neuron innervation of these cells [7, 29]. The glycinergic receptors on large DCN neurons have been shown to be contacted by neurons that are quiescent until excited [5]. The fact that the Gly-I cells are intrinsically silent and that no afferent glycinergic fibers have been demonstrated in the DCN suggests that the presynaptic terminals responsible for the glycinergic synaptic currents observed by Pedroarena and Kamphausen [5] would originate from local collaterals of the Gly-I cells rather than from the constitutively active Gly-A cells putatively co-releasing GABA and glycine. In contrast, presynaptic boutons containing both GABA and glycine have been demonstrated to be apposed to dendritic glycinergic synapses of DCN neurons even though the identity of postsynaptic cells was not examined [1]. This would suggest that the axons of Gly-A cells target dendritic rather than somatic membranes, if the Gly-A cells indeed co-express GABA and glycine, as our data suggests. Notably, our previous work [19] also provided evidence for local GABAergic neurons contacting dendritic rather than somatic synapses. Since the excitatory synapses are also known to reside mainly on DCN neuron dendrites [7], these presynaptic GABA/glycinergic neurons could be involved in modulating synaptic integration of glutamatergic inputs to the DCN.

As for the circuitry outside the DCN, the postsynaptic targets of the Gly-I axons projecting to the cerebellar cortex could not be identified even though several of the nucleocortical Gly-I axons were clearly seen to enter the granule cell layer. The only neurons expressing glycine receptors in most areas of the granule cell layer are Golgi cells, a proportion of which are also glycinergic [30, 31; but see also 32]. Golgi cells are known to receive glycinergic input from the intrinsically silent [32] Lugaro cell axons in the cerebellar molecular layer [33-35] but glycine receptors have also been found on the Golgi cell dendrites within granule cell layer. These glycine receptors on Golgi cell 
basal dendrites are reportedly not targeted by other Golgi cells [36, 37] and thus could be the targets of the nucleocortical Gly-I cells described here. As it is expected that most of long-range axons are cut in slice preparation, we cannot exclude the possibility that the axons would rise all the way through the granule cell layer and make synapses on Golgi cells in the molecular layer.

\section{Conclusions}

The present study shows that there are two morphologically and electrophysiologically distinct populations of putatively glycinergic neurons in the young adult lateral DCN, one of which seems to project towards the cerebellar cortex. While the precise targets of these projections as well as the demonstration of functional glycinergic synaptic responses involving the neuron types described here remain presently unverified in the absence of electron microscopy studies and paired electrophysiological recordings, the possible implications raised by the present study as well as the recent demonstration of glycinergic neurons of the fastigial DCN that project to the brainstem [21] demand for further studies of the role of glycinergic neurons of the DCN at the circuit and system level.

Acknowledgement We thank Drs. Hanns Ulrich Zeilhofer and Louis Scheurer for the kind gift of bacterial artificial chromosome transgenic mice in which glycinergic neurons express enhanced green fluorescent protein, Yuka Iwamoto for expert technical help with maintaining and genotyping mice, and all members of the Knöpfel Lab for helpful comments and suggestions. This work was supported by intramural funds provided by RIKEN.

Open Access This article is distributed under the terms of the Creative Commons Attribution Noncommercial License which permits any noncommercial use, distribution, and reproduction in any medium, provided the original author(s) and source are credited.

\section{References}

1. Chen S, Hillman DE (1993) Colocalization of neurotransmitters in the deep cerebellar nuclei. J Neurocytol 22:81-91

2. Zafra F, Aragón C, Giménez C (1997) Molecular biology of glycinergic neurotransmission. Mol Neurobiol 14:117-142

3. Rampon C, Luppi PH, Fort P, Peyron C, Jouvet M (1996) Distribution of glycine-immunoreactive cell bodies and fibers in the rat brain. Neuroscience 75:737-755

4. Kawa K (2003) Glycine receptors and glycinergic synaptic transmission in the deep cerebellar nuclei of the rat: a patchclamp study. J Neurophysiol 90:3490-3500

5. Pedroarena CM, Kamphausen S (2008) Glycinergic synaptic currents in the deep cerebellar nuclei. Neuropharmacology 54:784-795

6. Uusisaari M, Obata K, Knöpfel T (2007) Morphological and electrophysiological properties of GABAergic and non-GABAergic cells in the deep cerebellar nuclei. J Neurophysiol 97:901-911

7. Chan-Palay V (1977) Cerebellar dentate nucleus: organization, cytology and transmitters. Springer, New York
8. Sultan F, König T, Möck M, Thier P (2002) Quantitative organization of neurotransmitters in the deep cerebellar nuclei of the Lurcher mutant. J Comp Neurol 452:311-323

9. Bäurle J, Grüsser-Cornehls U (1997) Differential number of glycine- and GABA-immunopositive neurons and terminals in the deep cerebellar nuclei of normal and Purkinje cell degeneration mutant mice. J Comp Neurol 382(4):443-458

10. Zeilhofer HU, Studler B, Arabadzisz D, Schweizer C, Ahmadi S, Layh B et al (2005) Glycinergic neurons expressing enhanced green fluorescent protein in bacterial artificial chromosome transgenic mice. J Comp Neurol 482:123-141

11. Eulenburg V, Armsen W, Betz H, Gomeza J (2005) Glycine transporters: essential regulators of neurotransmission. Trends Biochem Sci 30:325-333

12. Poyatos I, Ponce J, Aragón C, Giménez C, Zafra F (1997) The glycine transporter GLYT2 is a reliable marker for glycineimmunoreactive neurons. Mol Brain Res 49:63-70

13. Tamamaki N, Yanagawa Y, Tomioka R, Miyazaki J, Obata K, Kaneko T (2003) Green fluorescent protein expression and colocalization with calretinin, parvalbumin, and somatostatin in the GAD67-GFP knock-in mouse. J Comp Neurol 467:60-79

14. Diez-Garcia J, Matsushita S, Mutoh H, Nakai J, Ohkura M, Yokoyama $J$ et al (2005) Activation of cerebellar parallel fibers monitored in transgenic mice expressing a fluorescent $\mathrm{Ca}^{2+}$ indicator protein. Eur J NeuroSci 22:627-635

15. LeDoux MS, Hurst DC, Lorden JF (1998) Single-unit activity of cerebellar nuclear cells in the awake genetically dystonic rat. Neuroscience 86:533-545

16. Rowland NC, Jaeger D (2005) Coding of tactile response properties in the rat deep cerebellar nuclei. J Neurophysiol 94:1236-1251

17. Pugh JR, Raman IM (2009) Nothing can be coincidence: synaptic inhibition and plasticity in the cerebellar nuclei. Trends Neurosci $32: 170-177$

18. Tanaka I, Ezure K (2004) Overall distribution of GLYT2 mRNAcontaining versus GAD67 mRNA-containing neurons and colocalization of both mRNAs in midbrain, pons, and cerebellum in rats. Neurosci Res 49:165-178

19. Uusisaari M, Knöpfel T (2008) GABAergic synaptic communication in the GABAergic and non-GABAergic cells in the deep cerebellar nuclei. Neuroscience 156:537-549

20. Bengtsson F, Hesslow G (2006) Cerebellar control of the inferior olive. Cerebellum 5:7-14

21. Bagnall MW, Zingg B, Sakatos A, Moghadam SH, Zeilhofer HU, Lac SD (2009) Glycinergic projection neurons of the cerebellum. J Neurosci 29:10104-10110

22. Tolbert DL, Bantli H, Bloedel JR (1976) Anatomical and physiological evidence for a cerebellar nucleo-cortical projection in the cat. Neuroscience 1:205-217

23. Angaut P, Compoint C, Buisseret-Delmas C, Batini C (1996) Synaptic connections of Purkinje cell axons with nucleocortical neurones in the cerebellar medial nucleus of the rat. Neurosci Res 26:345-348

24. Batini C, Buisseret-Delmas C, Compoint C, Daniel H (1989) The GABAergic neurones of the cerebellar nuclei in the rat: projections to the cerebellar cortex. Neurosci Lett 8(99):251-256

25. Batini C, Compoint C, Buisseret-Delmas C, Daniel H, Guegan M (1992) Cerebellar nuclei and the nucleocortical projections in the rat: retrograde tracing coupled to GABA and glutamate immunohistochemistry. J Comp Neurol 315:74-84

26. Provini L, Marcotti W, Morara S, Rosina A (1998) Somatotopic nucleocortical projections to the multiple somatosensory cerebellar maps. Neuroscience 83:1085-1104

27. De Zeeuw CI, Berrebi AS (1995) Postsynaptic targets of Purkinje cell terminals in the cerebellar and vestibular nuclei of the rat. Eur J NeuroSci 7:2322-2333 
28. Kolston J, Apps R, Trott JR (1995) A combined retrograde tracer and GABA-immunocytochemical study of the projection from nucleus interpositus posterior to the posterior lobe $\mathrm{C} 2$ zone of the cat cerebellum. Eur J NeuroSci 7:926-933

29. Hoebeek F, Khosrovani S, Witter L, De Zeeuw C (2008) Purkinje cell input to cerebellar nuclei in tottering: ultrastructure and physiology. Cerebellum 7:547-558

30. van den Pol A, Gorcs T (1988) Glycine and glycine receptor immunoreactivity in brain and spinal cord. J Neurosci 8:472-492

31. Dumoulin A, Triller A, Dieudonne S (2001) IPSC kinetics at identified GABAergic and mixed GABAergic and glycinergic synapses onto cerebellar Golgi cells. J Neurosci 21:60456057

32. Kaneda M, Farrant M, Cull-Candy SG (1995) Whole-cell and single-channel currents activated by GABA and glycine in granule cells of the rat cerebellum. J Physiol 485:419-435
33. Dean I, Robertson SJ, Edwards FA (2003) Serotonin drives a novel GABAergic synaptic current recorded in rat cerebellar Purkinje cells: a Lugaro cell to Purkinje cell synapse. J Neurosci 1 (23):4457-4469

34. Dieudonné S (1995) Glycinergic synaptic currents in Golgi cells of the rat cerebellum. Proc Natl Acad Sci U S A 92:1441-1445

35. D'Angelo E (2008) The critical role of Golgi cells in regulating spatio-temporal integration and plasticity at the cerebellum input stage. Front Neurosci 2:35-46

36. Dieudonne S, Dumoulin A (2000) Serotonin-driven long-range inhibitory connections in the cerebellar cortex. J Neurosci 20:1837-1848

37. Simat M, Ambrosetti L, Lardi-Studler B, Fritschy J (2007) GABAergic synaptogenesis marks the onset of differentiation of basket and stellate cells in mouse cerebellum. Eur J NeuroSci 26:2239-2256 